\section{OPEN}

SUBJECT AREAS:

EVOLUTIONARY

ECOLOGY

ENTOMOLOGY

Received

26 November 2013

Accepted

2 January 2014

Published

23 January 2014

Correspondence and requests for materials should be addressed to K.F.H. (khaynes@uky. edu)

\title{
Population variation in and selection for resistance to pyrethroid-neonicotinoid insecticides in the bed bug
}

\author{
Jennifer R. Gordon, Mark H. Goodman, Michael F. Potter \& Kenneth F. Haynes
}

Department of Entomology, University of Kentucky, Lexington, KY 40546-0091, USA.

Pyrethroid resistance in bed bugs, Cimex lectularius, has prompted a change to combination products that include a pyrethroid and a neonicotinoid. Ten populations of bed bugs were challenged with two combination products (Temprid SC ${ }^{\circledR}$ and Transport GHP ${ }^{\circledR}$ ). Susceptibility of these populations varied, with the correlated response of the two products indicating cross resistance. We imposed selection on three populations using label rate Temprid, and then reared progeny from unselected and selected strains. All selected strains were significantly less susceptible to Temprid SC than unselected strains. Temprid selected strains were also less susceptible to Transport. The pyrethroid component of Temprid showed a significantly higher $\mathrm{LD}_{50}$ in selected strains, but susceptibility to the neonicotinoid remained unchanged. Taken together these results indicate resistance to combination insecticides is present in field populations at levels that should be of concern, and that short-term selection affecting existing variance in susceptibility can quickly increase resistance.

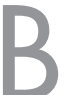

ed bugs, Cimex lectularius L., are hematophagous parasites that are resurging throughout the world ${ }^{1-7}$. These bugs became scarce during the second part of the twentieth century, likely because they were effectively controlled by DDT and other broad-spectrum insecticides. While infestations worldwide were declining, insecticide resistance was being reported ${ }^{8,9}$.

Insecticide resistance is a world-wide phenomenon that has been observed in hundreds of insect species of medical, urban and agricultural importance ${ }^{10-12}$. Recently, pyrethroid resistance has been found in populations of bed bugs ${ }^{13-17}$ and has been implicated as one of many factors in the current resurgence of these insects. There are only a few classes of insecticides with different modes of action approved for use against bed bugs ${ }^{18}$; thus, when pyrethroids fail, there are few options for rotation. Two classes of insecticide now commonly used to control bed bugs are pyrethroids (interfering with sodium ion channels ${ }^{19}$ ) and neonicotinoids (acting at nicotinic acetylcholine receptors ${ }^{20}$ ).

Recently, insecticidal products containing both a pyrethroid and a neonicotinoid have become available for bed bug control and are being widely used by pest management professionals ${ }^{21}$. Two such products are Temprid SC ${ }^{\circledR}$ ( $\beta$-cyfluthrin and imidacloprid) and Transport GHP ${ }^{\circledR}$ (bifenthrin and acetamiprid). Preliminary testing of these products has been promising, but evolution of resistance is a concern, particularly given that resistance to the pyrethroid component is common.

The purpose of this study was twofold. First, to gauge susceptibility to current combination products, residual bioassays were conducted using ten populations of bed bugs. Second, to determine the evolutionary response of three of these populations to a pyrethroid/neonicotinoid combination product, two hypotheses were tested: selection imposed by Temprid SC results in decreased susceptibility to this product that also translates into cross resistance (broadest sense) to Transport GHP (a second pyrethroid/neonicotinoid combination product); and both pyrethroid and neonicotinoid resistance are increased by selection with Temprid SC.

\section{Results}

Population survey of susceptibility. Susceptibility to both Temprid SC and Transport GHP varied among populations of bed bugs (Fig. 1). CIN10, FF1, LEX5, LEX7, LEX8, and RO1 were less susceptible to both products than CIN1, FD, LA1, and NY1 (Fig. 1; 1d-3d). After 1 d exposure to Temprid SC, RO1, LEX5, LEX7 and LEX8 were unaffected ( $0 \%$ mortality), and CIN10 and FF1 had less than 5\% mortality. This same exposure time resulted in intermediate mortality for two populations (CIN1 and NY1) and 100\% mortality for LA1 and FD. However, exposure to Transport GHP resulted in three populations with initially high mortality (CIN1, FD and 


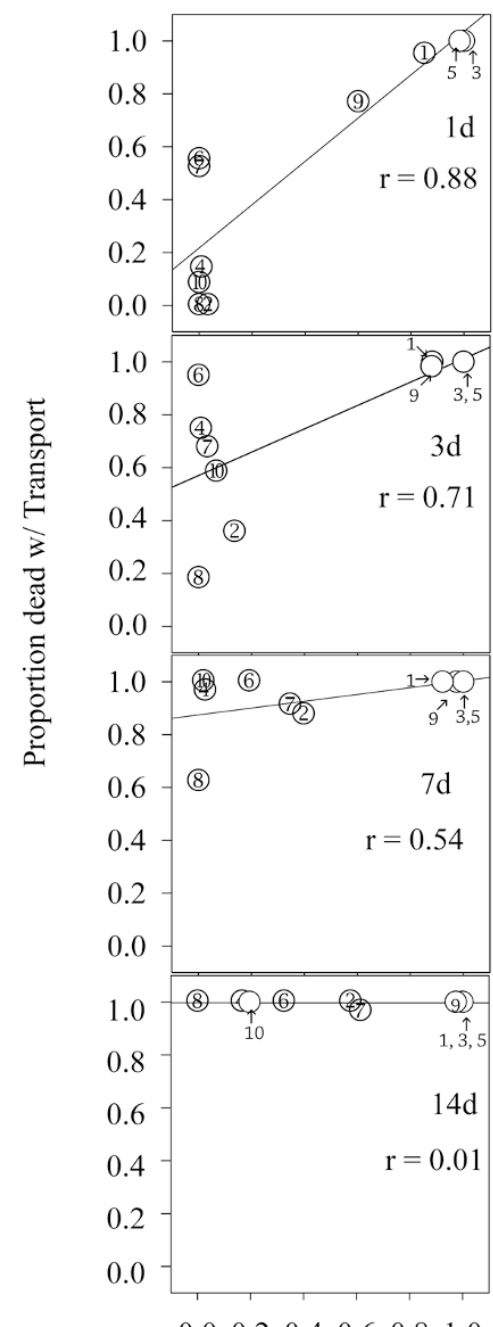

$\begin{array}{lllllll}0.0 & 0.2 & 0.4 & 0.6 & 0.8 & 1.0\end{array}$

Proportion dead w/ Temprid

Figure $1 \mid$ Regression between proportion of bed bugs killed by residues of Temprid SC and Transport GHP for ten populations (1 corresponds with CIN1, 2 corresponds with CIN10, 3 corresponds with FD, 4 corresponds with FF1, 5 corresponds with LA1, 6 corresponds with LEX5, 7 corresponds with LEX7, 8 corresponds with LEX8, 9 corresponds with NY1 and 10 corresponds with RO1) of bed bugs at 1 to $14 \mathrm{~d}$ of exposure. Data shown were corrected for control mortality.

LA1), five populations with intermediate mortality (FF1, LEX5, LEX7, NY1 and RO1), and only two populations with $0 \%$ mortality (CIN10 and LEX8). The correlation between susceptibility of these populations to Temprid SC and Transport GHP was initially high at $1 \mathrm{~d}(\mathrm{r}=0.88)$ but progressively dropped to its lowest value at $14 \mathrm{~d}(\mathrm{r}=0.01)$. The initially high correlation likely reflects the common mode of action of the active ingredients of the two products (both contain a pyrethroid and a neonicotinoid). The lack of correlation at $14 \mathrm{~d}$ was due to the convergence of all populations on $100 \%$ mortality with Transport GHP (all at $100 \%$ except LEX7 which had $96.5 \%$ mortality). After $14 \mathrm{~d}$ exposure to Temprid SC, CIN1, FD, and LA1 had 100\% mortality with NY1 at 97.5\%. At this same time, five other populations showed intermediate levels of mortality $(\mathrm{FF} 1=16.7 \%$; $\mathrm{RO} 1=19.6 \%$; LEX5 $=32.6 \%$; CIN10 $=57.6 \%$; LEX7 $=61.4 \%)$. Survival of LEX8 was not affected by Temprid SC (no mortality after 14 days).

The wide variation in susceptibility observed between populations could reflect independence of their evolutionary history of exposure to pyrethroids, neonicotinoids, or combinations of these active ingredients. FD and LA1 are susceptible to pyrethroids ${ }^{13}$. FD is a strain that has been maintained without insecticide exposure since the early $1970 \mathrm{~s}^{22}$. CIN1 was found to be highly resistant to deltamethrin and $\lambda$-cyhalothrin (pyrethroids) ${ }^{13}$ when it was first established as a laboratory colony but subsequently has reverted toward susceptibility (now only moderately resistant ${ }^{24}$ ). Synergist and RNAi studies indicate that CIN1 has P450 mediated enhanced metabolism of pyrethroids ${ }^{17,23,24}$. The reversion toward susceptibility could be due to a fitness cost from metabolic insecticide detoxification ${ }^{25}$. NY1 also had initially high levels of resistance to pyrethroids ${ }^{17}$, which have declined somewhat before the current study ${ }^{24}$. NY1 was initially resistant to deltamethrin via two $k d r$ target site mutations ${ }^{17}$ and P450-mediated enhanced metabolism ${ }^{24}$. All remaining populations were collected from the field after pyrethroid/neonicotinoid products became more widely used by the pest control industry for bed bugs. Thus their susceptibilities to these combination products could reflect a history of selection with either pyrethroids alone or in combination with neonicotinoids. A single action neonicotinoid product was not widely used for bed bug management before this study was initiated.

Selection experiment. Because of initial population variation in susceptibility, we exposed CIN1, LA1, and NY1 for different times to Temprid SC to impose selection (kill approximately 80\%; Supplemental Fig. S1). The actual mean mortality achieved by exposing CIN1, LA1, and NY1 for $1 \mathrm{~h}, 0.1 \mathrm{~h}$, and $19 \mathrm{~h}$ were 83.6 (s.e.m. \pm 1.0$), 81.7$ (s.e.m. \pm 0.5 ), 82.4 (s.e.m. \pm 3.9 )\% for females and 81.3 (s.e.m. \pm 1.3 ), 85.2 (s.e.m. \pm 3.8 ), and 93.5 (s.e.m. \pm 1.3$) \%$ for males, respectively (mean of two replicates, see Table 1). We observed decreased susceptibility to Temprid SC within one generation in each strain. Mortality at the exposure time to the label rate material that causes $80 \%$ mortality in the population $\left(\mathrm{ET}_{80}\right)$ decreased significantly as a result of selection in CIN1 $\left(\mathrm{G}^{2}\right.$ $=146 ; \mathrm{df}=1 ; \mathrm{p}<0.001$ ), LA1 (Fig. $2 ; \mathrm{G}^{2}=101 ; \mathrm{df}=1 ; \mathrm{p}<$ $0.001)$, and NY1 ( $\mathrm{G}^{2}=71 ; \mathrm{df}=1 ; \mathrm{p}<0.001$; Table 2$)$. When these populations were tested with an alternate combination product, Transport GHP (using the same $\mathrm{ET}_{80}$ s for Temprid SC) mortality decreased as a response to selection imposed with Temprid SC for CIN1 $\left(\mathrm{G}^{2}=169 ; \mathrm{df}=1 ; \mathrm{p}<0.001\right)$ and LA1 (Fig. 3; $\mathrm{G}^{2}=54 ; \mathrm{df}=1$; $\mathrm{p}<0.001$; Table 2) indicating cross resistance between combination products utilizing alternate active ingredients. Because there were insufficient numbers of bed bugs in the first offspring generation, NY1 was not evaluated for cross resistance between Temprid SC and Transport GHP.

Susceptibility to $\boldsymbol{\beta}$-Cyfluthrin and Imidacloprid. The evolution that we observed as a result of selection with combination products could be due to changes in susceptibility to either

Table 1 | Percentage mortality realized when each strain was exposed to dry residues of the label rate $(0.075 \%$ a.i.) of Temprid SC application for an exposure time targeting $80 \%$ mortality $\left(E_{80}\right)$, and thus expected to impose strong selection

\begin{tabular}{lccc} 
Strain & Replicate & \multicolumn{2}{c}{$\%$ Mortality $^{a}$ (number treated) } \\
\hline \multirow{2}{*}{ LA1 } & Rep 1 & $82.2(370)$ & \% \\
& Rep 2 & $81.2(410)$ & $89.0(10)$ \\
CIN1 & Rep 1 & $84.5(360)$ & $82.5(120)$ \\
& Rep2 & $82.6(390)$ & $80.0(100)$ \\
NY1 & Rep 1 & $78.5(492)$ & $92.2(180)$ \\
& Rep 2 & $86.3(490)$ & $94.8(210)$ \\
\hline
\end{tabular}

${ }^{a}$ Groups of bugs were treated with the strain respective ET 80 (LA1 0.1 h, CIN1 1 h, NY1 19 h; Supplemental Fig. S1).

${ }^{b}$ More females were exposed than males to achieve a sex ratio of $5: 2$ : : o' to reduce the deleterious impact of excessive traumatic matings. 


\section{Temprid}

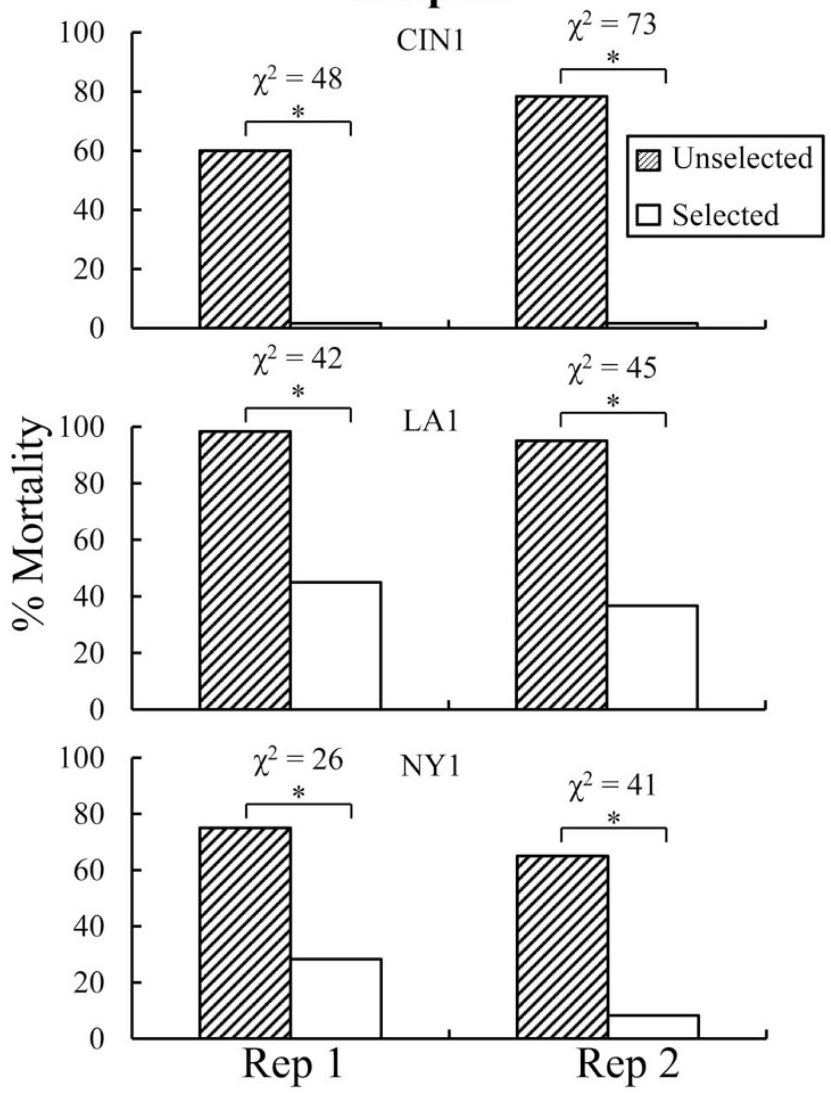

Figure 2 Susceptibility to Temprid SC in selected and unselected lines from CIN1, LA1, and NY1. In the parental generation adult bed bugs were exposed to residues of Temprid SC for intervals expected to kill $80 \%$ at $24 \mathrm{~h}$ after exposure for selected lines. Unselected lines were treated in the same way but without insecticide exposure. In each replicate of each strain there was a significant decrease (each $\chi^{2}$ analysis had $1 \mathrm{df}$ ) in offspring mortality from the same exposure as parents received (see text for log linear analysis).

insecticidal component or to both. Probit regression lines for all three selected strains compared to unselected strains were shifted to higher doses for $\beta$-cyfluthrin but not for imidacloprid (Fig. 4 and Fig. 5). All three populations showed a significant increase in the dose that kills $50 \%$ of the population $\left(\mathrm{LD}_{50}\right)$ after selection compared to unselected

\section{Transport}

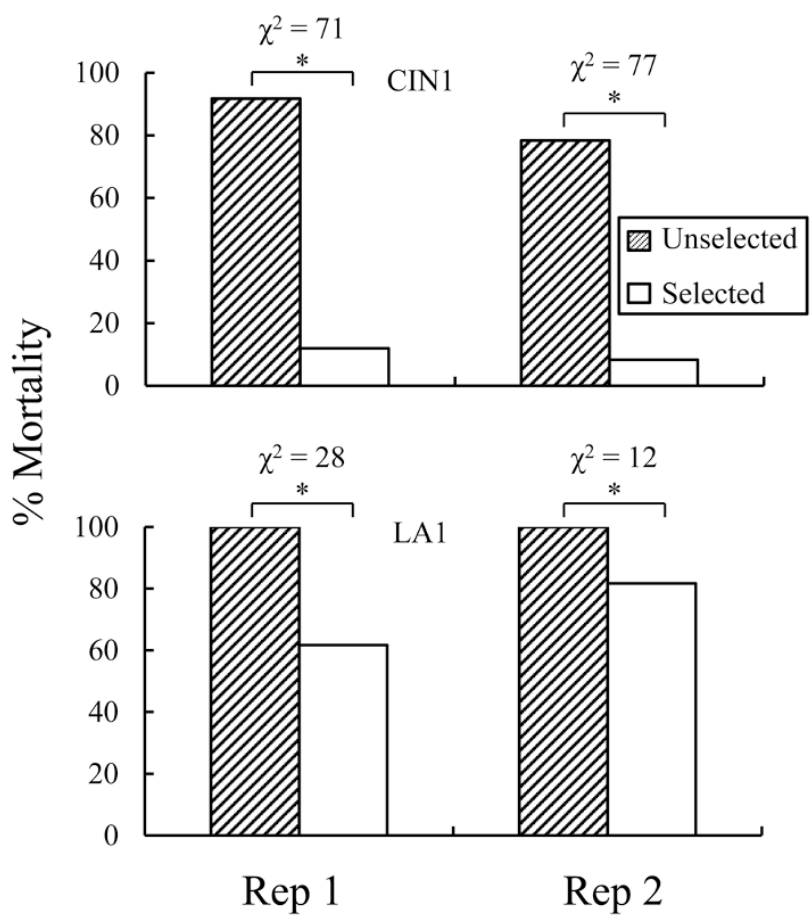

Figure $3 \mid$ Susceptibility to Transport GHP in selected and unselected lines from CIN1 and LA1 showing cross resistance to Temprid SC. In the parental generation, adult bed bugs were exposed to residues of Temprid SC for intervals expected to kill $80 \%$ at $24 \mathrm{~h}$ after exposure for selected lines. Unselected lines were treated in the same way but without insecticide exposure. NY1 was not evaluated because of insufficient numbers of test insects. In each replicate of both populations, there was a significant decrease (each $\chi^{2}$ analysis had $1 \mathrm{df}$ ) in offspring mortality from Transport GHP exposure (see text for log linear analysis).

strains for $\beta$-cyfluthrin (Table 3 ). Susceptibility to imidacloprid only changed significantly in LA1 and NY1 (Table 3); however, $\mathrm{LD}_{50}$ values were relatively unaffected especially compared to $\beta$ cyfluthrin. The contrast between selected and unselected regression lines for all three populations significantly departed from parallelism for $\beta$-cyfluthrin but remained unchanged for imidacloprid (Table 3 ). The departure from parallelism results in the convergence of regression lines for $\mathrm{CIN} 1$ and $\mathrm{NY} 1$ and a divergence for LA1 at

Table 2 | Mortality of unselected and selected offspring to Temprid SC and Transport GHP using an exposure time estimated to kill $80 \%$ of the initial population prior to selection

\begin{tabular}{|c|c|c|c|c|c|}
\hline Insecticide & Strain & Treatment & $\%$ Mortality (土s.e.m.) $)^{a}$ & $\mathrm{n}^{b}$ & $\mathrm{G}^{2}(\mathrm{df} ; \mathrm{p} \text {-value })^{c}$ \\
\hline \multirow[t]{5}{*}{ Temprid SC } & CIN1 & Unselected & $69.2( \pm 9.2)$ & 120 & $146(1,<0.001)^{*}$ \\
\hline & CIN1 & Selected & $1.6( \pm 0.0)$ & 120 & \\
\hline & LAl & Unselected & $96.7( \pm 1.7)$ & 120 & $101(1,<0.001)^{*}$ \\
\hline & NY1 & Unselected & $70.0( \pm 5.0)$ & 120 & $71(1 ;<0.001)^{*}$ \\
\hline & NY1 & Selected & $18.3( \pm 10.0)$ & 120 & \\
\hline \multirow[t]{2}{*}{ Transport GHP } & CIN1 & Unselected & $85.0( \pm 6.7)$ & 120 & $169(1 ; 0.001)^{*}$ \\
\hline & LAl & Selected & $71.7( \pm 10.0)$ & 120 & \\
\hline
\end{tabular}




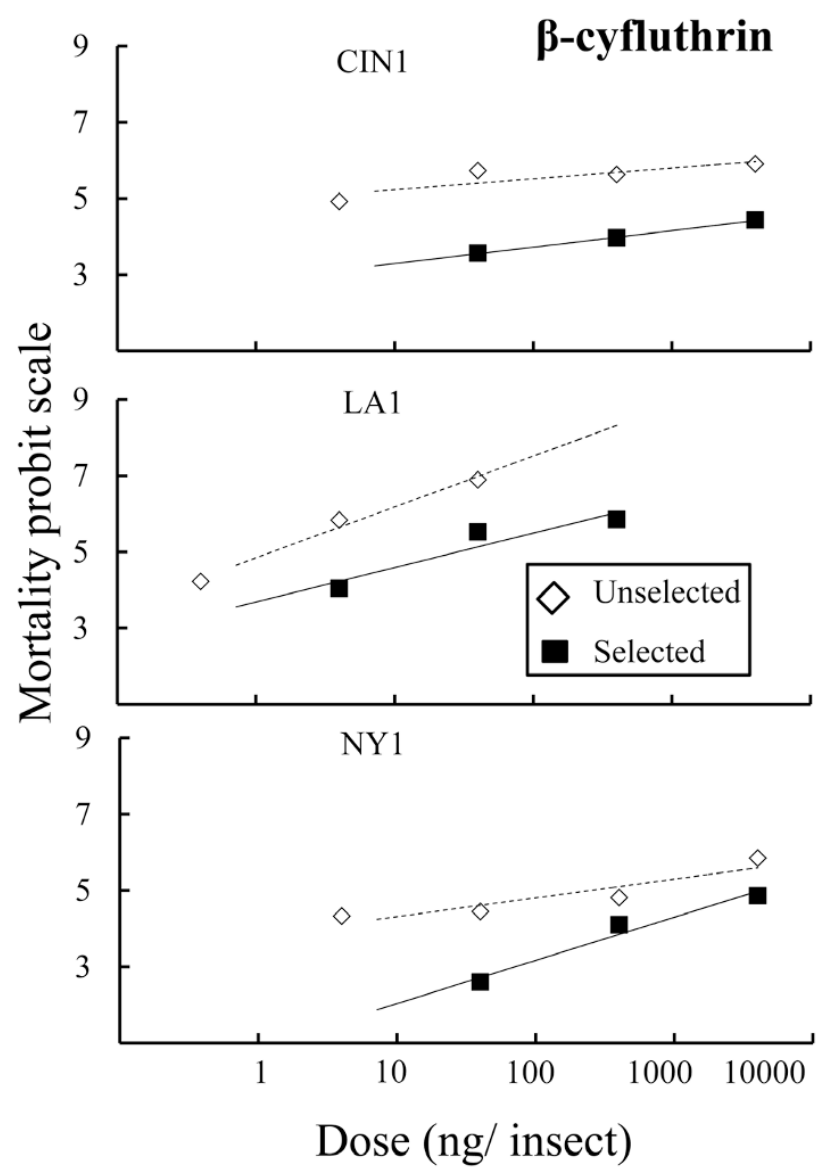

Figure $4 \mid$ Probit regression data for the relationship between dose of $\beta$ cyfluthrin and mortality at $\mathbf{2 4} \mathrm{h}$ for topical bioassays. Open diamonds and dotted lines represent unselected strains; whereas solid squares and solid lines represent selected strains.

higher doses (Fig. 4). This result suggests that after selection with Temprid SC the distribution of susceptibility to $\beta$-cyfluthrin changed within all populations but not to imidacloprid and further indicates that selection occurs due to the pyrethroid but not the neonicotinoid.

\section{Discussion}

There are few effective classes of insecticides available today for bed bug management, in part because the predominant use of pyrethroid-based products has selected for resistance in many populations of bed bugs ${ }^{5,26}$. Phantom ${ }^{\circledR}$ (chlorfenapyr), a pyrrole insecticide acting on oxidative phosphorylation rather than sodium channels of nerve cells, killed pyrethroid-resistant bed bugs ${ }^{27}$, but the relatively slow action of chlorfenapyr has prompted continued interest in alternatives. Dual action products containing both pyrethroids and neonicotinoids are now favored by a majority of pest management professionals ${ }^{28}$. Given the prevalence of pyrethroid resistance in field populations, resistance to pyrethroid/neonicotinoid combination products is a concern, especially considering the diverse toxicological defenses inherent in this insect ${ }^{24}$. All populations of bed bugs collected in the field during the last two years were less susceptible to a pyrethroid/neonicotinoid combination than our longer maintained laboratory colonies (Table 4). This included one strain (LEX8) that showed no mortality after resting on freshly dried residues of Temprid SC for two weeks and three other populations with less than $50 \%$ mortality at that time. In addition to a survey of susceptibility among laboratory populations, we also investigated the potential of three of our established populations to evolve resistance to combination products under laboratory conditions.

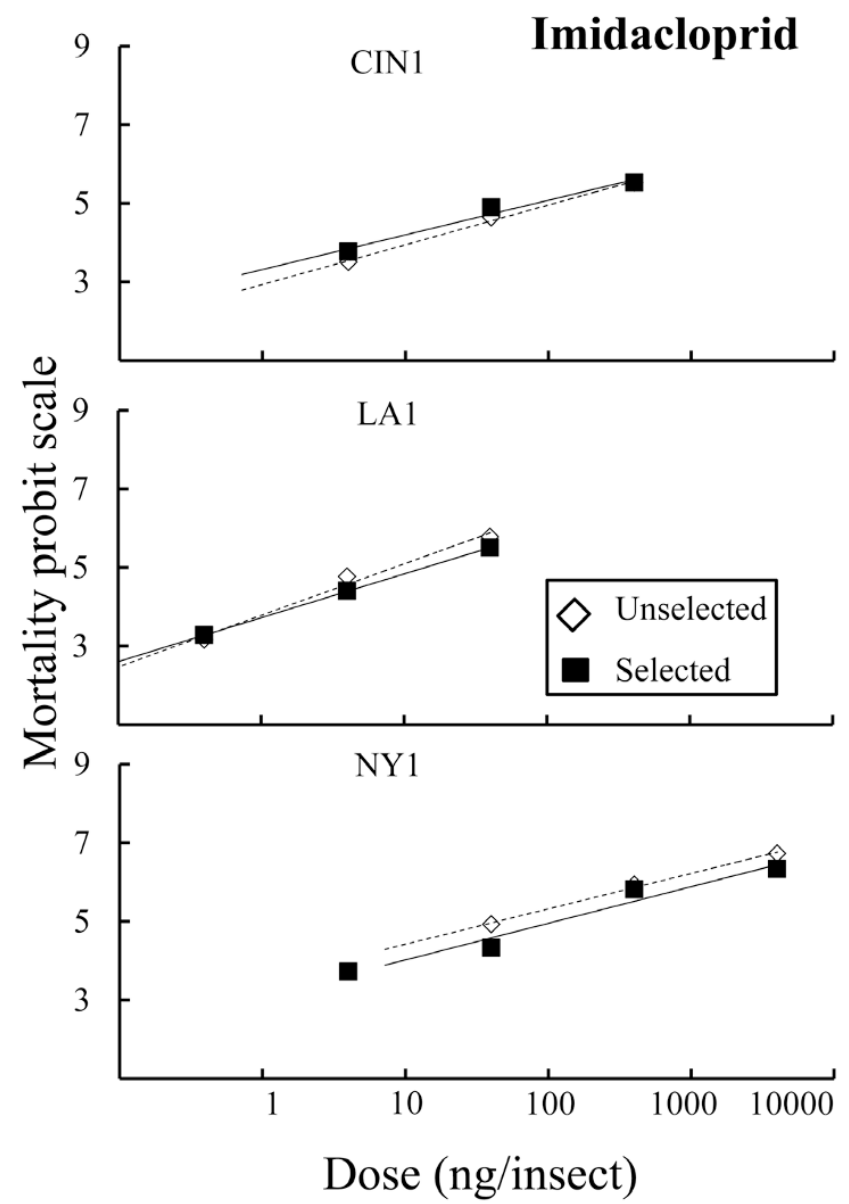

Figure $5 \mid$ Probit regression data for the relationship between dose of imidacloprid and mortality at $\mathbf{2 4} \mathrm{h}$ for topical bioassays. Open diamonds and dotted lines represent unselected strains; whereas solid squares and solid lines represent selected strains.

Three populations of bed bugs (CIN1, LA1, NY1) responded to selection with Temprid SC within one generation. This is the first laboratory documented case of decreased susceptibility as a result of selection to a pyrethroid/neonicotinoid product in bed bugs. In the absence of insecticide exposure, CIN1 and NY1 had been evolving away from the high levels of pyrethroid resistance that were initially observed $^{13,17,24}$, perhaps indicating a tradeoff between insecticide resistance and some unknown life history characteristics. However, if genes for pyrethroid resistance were still common in these two populations, then selection could have a large effect quickly.

Cross resistance between insecticides with the same mode of action is expected and can result in the loss of an entire class of insecticides ${ }^{12}$. Here we show that cross resistance between two pyrethroid/neonicotinoid combination products with different active constituents occurs as would be expected since collectively these constituents target the same neuronal sites (i.e., pyrethroids target sodium channels on neurons and neonicotinoids target nicotinic acetylcholine receptors). In addition, cross resistance between pyrethroids and neonicotinoids has been documented in other insects ${ }^{29,30}$ and is likely due to shared mechanisms of detoxification. For example, the cytochrome P450 class of enzymes is known to detoxify both pyrethroids and neonicotinoids ${ }^{31,32}$. However, our results indicate that the change in susceptibility to Temprid SC is largely effected by a change in pyrethroid susceptibility.

Monitoring insecticide susceptibility and managing insecticide resistance are core principles of successful integrated pest management ${ }^{33-35}$. The practicality of some of the principles developed in 


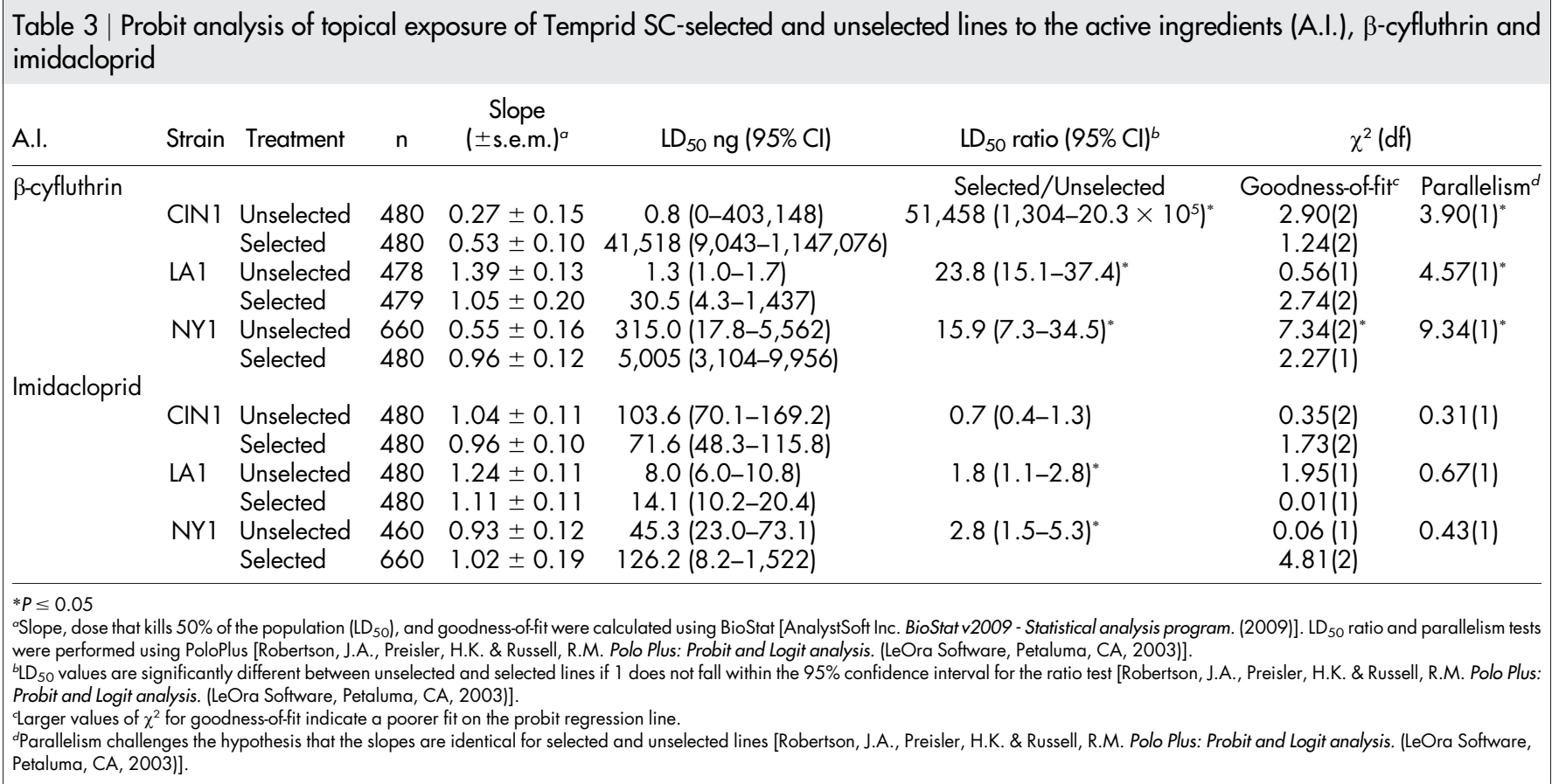

open agricultural systems are limited by zero tolerance for bed bug populations, lack of gene flow between susceptible and resistant populations, and limited number of available insecticides with different modes of action for rotation. In contrast, the return to susceptibility that has been noted in laboratory-reared populations indicates that resistance conferring genes may not be fixed within all populations of bed bugs. Selection of control tactics based on population specific resistance profiles could make bed bug management more effective. Genetically varied populations make it unlikely that one universally favored product will be an enduring solution. Prescription treatments would need to be implemented on a building by building basis, because within building genetic diversity is low (indicating a genetically restricted founding effect) and between building diversity is high even within a small geographic location ${ }^{36}$. While not always economically feasible, use of alternative approaches such as whole building heat treatments or fumigation could be particularly helpful when insecticide resistance is indicated. Employing multiple tactics (heat, cold, vacuuming, bed encasement, etc.) along with vigilance and early detection may be the best way to mitigate resistance within populations of bed bugs.

\section{Methods}

Insects. Ten populations of bed bugs were used in this study (Table 4). Insects were housed in incubators away from any insecticide exposure at $26.7^{\circ} \mathrm{C}, 65 \pm 5 \% \mathrm{RH}$, and a photoperiod of $14: 10(\mathrm{~L}: \mathrm{D}) \mathrm{h}$. All bed bugs were fed weekly on warmed defibrinated rabbit blood (Quad Five, Ryegate, MT) through a parafilm membrane ${ }^{37}$.

Population survey of susceptibility. A residual bioassay ${ }^{13}$ was used to survey susceptibility of 10 bed bug populations to Temprid SC (Bayer, Research Triangle Park, NC) and Transport GHP (FMC, Philadelphia, PA). Individual wells of a 24-well cell culture plate (Costar, Corning, NY) were lined with filter paper disks (Whatman \#2, cut to $1.7 \mathrm{~cm}$ diam.). Label rate solutions (Temprid SC $0.075 \%$ a.i and Transport GHP $0.11 \%$ a.i.) were made by diluting the concentrated insecticide in water. Next, $50 \mu \mathrm{L}$ of each solution was pipetted onto filter papers fitted into individual wells, and then allowed to dry completely before bugs were placed on the surface. Mortality was scored after 1, 2, 3, 7 and $14 \mathrm{~d}$ continuous exposure to the treated filter papers. Six groups of ten bugs (60 individuals; Table 4) were used for each strain of bed bugs and treatment (Temprid, Transport or water), with the exceptions of NY1, CIN1 and FF1 (all strains and treatments utilized 120 bugs) and the LEX7 and LEX8 Transport

\begin{tabular}{|c|c|c|c|c|c|}
\hline Name & City & Collection Date & Pyrethroid Resistance $^{a}$ & Temprid SC ${ }^{\circledR}(\mathrm{n})^{b}$ & Transport GHP® $(n)^{c}$ \\
\hline CIN10 & Cincinnati, $\mathrm{OH}$ & 2012 & Highly resistant & $57.6(60)$ & $100(60)$ \\
\hline FD & Fort Dix, NJ & $<1974$ & Susceptible ${ }^{13}$ & $100(60)$ & $100(60)$ \\
\hline LEX5 & Lexington, $K Y$ & 2011 & Unknown & $32.6(60)$ & $100(60)$ \\
\hline LEX7 & Lexington, $\mathrm{KY}$ & 2012 & Highly resistant & $61.4(60)$ & $96.5(59)$ \\
\hline LEX8 & Lexington, $\mathrm{KY}$ & 2012 & Unknown & $0.0(60)$ & $100(51)$ \\
\hline NY1 & New York, NY & 2007 & $\begin{array}{l}\text { Initially highly resistant }{ }^{17} \text {, now moderately } \\
\text { resistant }^{24}\end{array}$ & $97.5(120)$ & $100(120)$ \\
\hline RO1 & Royal Oaks, Ml & 2012 & Unknown & $19.6(60)$ & $100(60)$ \\
\hline
\end{tabular}

aPyrethroid resistance categorization using a residual bioassay and discriminating dosage of deltamethrin $(0.6 \%)$. Populations were considered susceptible if mortality was $>95 \%$, moderately resistant if mortality was $<50 \%$, and highly resistant if mortality was $<5 \%$.

bPercent mortality of adult bugs after 14 days of exposure to label rate Temprid SC.

cPercent mortality of adult bugs after 14 days of exposure to label rate Transport GHP. 
experiments ( $\mathrm{n}=59 ; \mathrm{n}=51$ respectively). Insects were classified as dead if they showed no movement or were unable to right themselves within $15 \mathrm{~s}$ of being inverted with soft forceps. Abbott's formula ${ }^{38}$ was used to correct for control mortality.

Selection experiment. A residual deposit bioassay was used to select three strains and determine susceptibility of offspring at strain specific $\mathrm{ET}_{80} \mathrm{~s}$. Filter paper disks (Whatman \#2; $4.25 \mathrm{~cm}$ diam.) were treated with Temprid SC $(0.075 \%)$. This insecticide was applied until the paper was uniformly wetted using a fine mist sprayer (ProChemical and Dye, Somerset, MA). A second series of disks were handled similarly but treated with water to serve as a control. Disks were allowed to dry overnight. Dry disks were placed into 6-well cell culture plates (Costar; Corning, NY) with the treated surface facing up. Individuals of each strain were exposed for strainspecific exposure times (LA1 0.1 h, CIN1 1 h, NY1 19 h). Because $80 \%$ mortality was expected, large numbers of females and males from each strain were used to start selected lines (Table 1). After this exposure, bugs were removed from the treated surface and placed individually in wells of a 24 -well plate lined with untreated filter paper. Mortality was scored $24 \mathrm{~h}$ after removal from treated substrates. To serve as a control, a second group of 100 females and 40 males per strain and replicate were exposed for the same amount of time to filter papers saturated with water. Two independent pairs of selected and unselected groups were created for each laboratory population (CIN1, LA1 and NY1). After initial selection, F1 and F2 progeny were used for all subsequent experiments and received no further selection. For both selected and unselected groups, survivors were removed after $24 \mathrm{~h}$ and placed in feeders ( $75 \mathrm{ml}$ plastic jars with organza covered lids; Consolidated Plastics, Stow, $\mathrm{OH}$ ) at a sex ratio of 5 females to 2 males (to reduce detrimental effects on females caused by traumatic insemination). Parental females were allowed to oviposit on blotter paper in the feeder. Adults were transferred to a new feeder weekly leaving a group of 0 to 7 day-old eggs behind.

Offspring were reared to the adult stage using the same methods described earlier ${ }^{37}$. These offspring were used in bioassays to measure susceptibility to Temprid SC and cross resistance to Transport GHP using the same residual assay as described above for selection with Temprid SC at the established $\mathrm{ET}_{80} \mathrm{~S}$.

Susceptibility to $\beta$-cyfluthrin and imidacloprid. Adults from the F2 generation were used to evaluate susceptibility to imidacloprid and $\beta$-cyfluthrin (technical grade; 99.5\% purity, Chem Services), the active ingredients of Temprid SC. Because numbers of bed bugs from the NY1 replicate two were adequate, the F1 generation was used. Topical bioassays were performed using doses ranging from 0.4 to $4000 \mathrm{ng} / \mathrm{insect}$ of either $\beta$-cyfluthrin or imidacloprid dissolved in acetone that was then applied to individual bed bugs with a repeating dispenser (Hamilton, Reno, NV). An aliquot $(0.5 \mu \mathrm{L})$ of a single insecticide dose was applied to the abdomens of equal numbers of males and females housed in individual wells of a 24 -well plate. For each dose, between 60 and 180 individuals were used. Bugs treated with acetone served as a control. Mortality was observed at $24 \mathrm{~h}$. No control mortality was observed.

Data analysis. Correlation analysis was used to examine the relationship between Temprid SC and Transport GHP susceptibility in the ten tested populations [Analytical Software. Statistix 8.0 for Windows. Tallahassee, FL (2003)]. The dependence of mortality on selection was explored using a log-linear analysis with treatment (selected vs. unselected), mortality (dead vs. alive), and replicate as three dimensions of a contingency table ${ }^{39}$. Individual replicates from the selection experiment were analyzed using $\chi^{2}$ analysis [Analytical Software. Statistix 8.0 for Windows. (Tallahassee, FL, 2003)]. Probit analysis was used for analysis of topical bioassays [AnalystSoft Inc. BioStat v2009 - Statistical analysis program. (2009)]. The hypotheses that test if strains are identical in terms of slope, intercept and $\mathrm{LD}_{50}$ were evaluated using Polo Plus software [Robertson, J.A., Preisler, H.K. \& Russell, R.M. Polo Plus: Probit and Logit analysis. (LeOra Software, Petaluma, CA, 2003)]. LD $_{50}$ values are significantly different between unselected and selected lines if the $95 \%$ confidence interval for their ratio does not include the ratio of 1 .

1. Doggett, S. L. \& Russell, R. C. The resurgence of bed bugs, Cimex spp. (Hemiptera: Cimicidae) in Australia. Paper presented at Sixth International Conference on Urban Pests, Budapest, Hungary. Hungary: OOK-Press Kft. (2008).

2. Kilpinen, O., Kristensen, M. \& Jensen, K. M. Resistance differences between chlorpyrifos and synthetic pyrethroids in Cimex lectularius population from Denmark. Parasitol. Res. 109, 1461-1464 (2011).

3. Mumcuoglu, K. Y. \& Shalom, U. Questionnaire survey of common bedbug (Cimex lectularius) infestations of Israel. Israel J. Entomol. 40, 1-10 (2010).

4. Omudu, E. A. \& Kuse, C. N. Bedbug infestation and its control practices in Gbajimba: a rural settlement in Benue state, Nigeria. J. Vector Borne Dis. 47, 222-227 (2010).

5. Potter, M. F., Rosenberg, B. \& Henriksen, M. Bugs without borders: Defining the global bed bug resurgence. Pest World. Sept./Oct., 8-20 (2010).

6. Bencheton, A. L. et al. Resurgence of bedbugs in southern France: A local problem or the tip of the iceberg? J. Eur. Acad. Dermatol. Venereol. 25, 599-602 (2011).

7. Tawatsin, A. et al. Insecticide resistance in bedbugs in Thailand and laboratory evaluation of insecticides for the control of Cimex hemipterus and Cimex lectularius (Hemiptera: Cimicidae). J. Med. Entomol. 48, 1023-1030 (2011).

8. Busvine, J. R. Insecticide resistance in bed bugs. Bull. World Health Organ. 19, 1041-1052 (1958)
9. Mallis, A. \& Miller, A. C. Prolonged resistance in the house fly and bed bug. J. Econ. Entomol. 57, 608-609 (1964).

10. Melander, A. L. Can insects become resistant to sprays? J. Econ. Entomol. 7, 167-172 (1914)

11. Forgash, A. J. History, evolution and consequences of insecticide resistance. Pestic. Biochem. Physiol. 22, 178-186 (1984).

12. Hemmingway, J. \& Ranson, H. Insecticide resistance in insect vectors of human disease. Annu. Rev. Entomol. 45, 371-391 (2000).

13. Romero, A., Potter, M. F., Potter, D. A. \& Haynes, K. F. Insecticide resistance in the bed bug: A factor in the pest's sudden resurgence? J. Med. Entomol. 44, 175-178 (2007).

14. Steelman, C. D. et al. Susceptibility of the bed bug Cimex lectularius L. (Heteroptera: Cimicidae) collected in poultry production facilities to selected insecticides. J. Agric. Urban. Entomol. 25, 41-51 (2008).

15. Yoon, K. S. et al. Biochemical and molecular analysis of deltamethrin resistance in the common bed bug (Hemiptera: Cimicidae). J. Med. Entomol. 45, 1092-1101 (2008).

16. Mamidala, P., Jones, S. C. \& Mittapalli, O. Metabolic resistance in bed bugs. Insects. 2, 36-48 (2011).

17. Zhu, F. et al. Widespread distribution of knockdown resistance mutations in the bed bug, Cimex lectularius (Hemiptera: Cimicidae), populations in the United States. Arch. Ins. Biochem. Physiol. 73, 245-257 (2010).

18. Davies, T. G. E., Field, L. M. \& Williams, M. S. The re-emergence of the bed bug as a nuisance pest: Implications of resistance to the pyrethroid insecticides. Med. Vet. Entomol. 26, 241-254 (2012).

19. Soderlund, D. M. \& Bloomquist, J. R. Neurotoxic action of pyrethroids. Ann. Rev. Entomol. 34, 77-96 (1989).

20. Tomizawa, M. \& Casida, J. E. Neonicotinoid insecticide toxicology: Mechanisms of selective action. Ann. Rev. Pharmacol. Toxicol. 45, 247-268 (2005)

21. Potter, M. F., Haynes, K. F., Gordon, J. R., Hardebeck, E. \& Wickemeyer, W. Dual action bed bug killers. Pest Control Technol. 40, 62-68, 75-76, (2012).

22. Bartley, J. \& Harlan, H. Bed bug infestation: Its control and management. Military Med. 139, 884-886 (1974).

23. Romero, A., Potter, M. F. \& Haynes, K. F. Evaluation of piperonyl butoxide as a deltamethrin synergist for pyrethroid-resistant bed bugs. J. Econ. Entomol. 102 2310-2315 (2009).

24. Zhu, F., Gujar, H., Gordon, J. R., Haynes, K. F., Potter, M. F. \& Palli, S. R. Bed bugs evolved unique adaptive strategy to resist pyrethroid insecticides. Sci. Rep. 3, 1456; DOI:10.1038/srep01456 (2013).

25. Kliot, A. \& Ghanim, M. Fitness costs associated with insecticide resistance. Pest Manag. Sci. 68, 1431-1437 (2012).

26. Potter, M. F., Haynes, K. F., Henriksen, M. \& Rosenberg, B. The 2011 bed bugs without borders survey. Pest World. Nov./Dec., 4-15 (2011).

27. Romero, A., Potter, M. F. \& Haynes, K. F. Evaluation of chlorfenapyr for control of the bed bug, Cimex lectularius L. Pest Manag. Sci. 66, 1243-1248 (2010).

28. Potter, M. F., Haynes, K. F., Fredericks, J. \& Henriksen, M. Bed bug nation: Are we making any progress? Pest World. Sept./Oct., 4-11 (2013).

29. Liu, N. \& Yue, X. Insecticide resistance and cross resistance in the house fly (Diptera: Muscidae). J. Econ. Entomol. 93, 1269-1275 (2000).

30. Basit, M., Sayyed, A. H., Saleem, M. A. \& Saeed, S. Cross resistance, inheritance and stability of resistance to acetamiprid in cotton whitefly, Bemisia tabaci Genn (Hemiptera: Aleyrodidae). Crop Protection. 30, 705-712 (2011).

31. Scott, J. Cytochromes P450 and insecticide resistance. Ins. Biochem. Molec. Biol. 29, 757-777 (1999)

32. Nauen, R. \& Denholm, I. Resistance of insect pests to neonicotinoid insecticides: Current status and future prospects. Arch. Ins. Biochem. Physiol. 58, 200-215 (2005).

33. Georghiou, G. P. Principles of insecticide resistance management. Phytoprotection. 75, 51-59 (1994).

34. Bennett, G. W., Owens, J. M. \& Corrigan, R. M. Truman's Scientific Guide to Pest Management Operations. (Purdue University Press, West Lafayette, Indiana, 2003).

35. Onstad, D. W. Major issues in insect resistance management. Insecticide Resistance Management. (ed.) Onstad, D. W. (Elsevier, San Diego, CA 2008).

36. Booth, W. et al. Molecular markers reveal infestation dynamics of the bed bug (Hemiptera: Cimicidae) within apartment buildings. J. Med. Entomol. 49, 535-546 (2012)

37. Montes, C., Cuadrillero, C. \& Vilella, D. Maintenance of a laboratory colony of Cimex lectularius (Hemiptera: Cimicidae) using an artificial feeding technique. J. Med. Entomol. 39, 675-679 (2002).

38. Abbott, W. S. A method of computing the effectiveness of an insecticide. J. Econ Entomol. 18, 265-267 (1925).

39. Sokal, R. R. \& Rohlf, F. J. Biometry. (W.H. Freeman, San Francisco, CA, 1981).

\section{Acknowledgments}

We thank Shelby Stamper, Reshma Anilkumar and Scott Bessin for technical assistance. Rollins, Inc., provided ongoing funding of the University of Kentucky's bed bug research J.R.G. was partly supported by an Urban Entomology Fellowship from the Department of Entomology, University of Kentucky. This is paper no. 13-08-102 of the Kentucky Agricultural Experiment Station. 


\section{Author contributions}

J.R.G. and K.F.H. wrote the manuscript. J.R.G. and K.F.H. prepared all figures and tables. J.R.G. and M.H.G. reared all strains of bed bugs. M.F.P. and K.F.H. contributed reagents/ materials/analysis tools. J.R.G. performed all experiments. J.R.G., K.F.H. and M.F.P. were responsible for developing the ideas contained in the manuscript. All authors reviewed the manuscript.

\section{Additional information}

Supplementary information accompanies this paper at http://www.nature.com/ scientificreports
Competing financial interests: The authors declare no competing financial interests.

How to cite this article: Gordon, J.R., Goodman, M.H., Potter, M.F. \& Haynes, K.F. Population variation in and selection for resistance to pyrethroid-neonicotinoid insecticides in the bed bug. Sci. Rep. 4, 3836; DOI:10.1038/srep03836 (2014).

\section{(c) (i) (2) This work is licensed under a Creative Commons Attribution-}

Br ${ }_{\text {NA }}$ NonCommercial-ShareAlike 3.0 Unported license. To view a copy of this license, visit http://creativecommons.org/licenses/by-nc-sa/3.0 